\title{
Global features of the Alcanivorax borkumensis SK2 genome
}

Oleg N. Reva, ${ }^{1,3}$, Peter F. Hallin ${ }^{2}$, Hanni Willenbrock, ${ }^{2}$ Thomas Sicheritz-Ponten, ${ }^{2}$ Burkhard Tümmler ${ }^{1}$ and David W. Ussery ${ }^{2}$

1 Klinische Forschergruppe, OE6711, Medizinische Hochschule Hannover, CarlNeuberg-Strasse 1 D-30625 Hannover, Germany.

2 Center for Biological Sequence Analysis, Technical University of Denmark, Lyngby, Denmark.

3 Biochemistry Department, University of Pretoria, Lynnwood Road, Hillcrest, 0002 Pretoria, South Africa.

\section{Summary}

The global feature of the completely sequenced Alcanivorax borkumensis SK2 type strain chromosome is its symmetry and homogeneity. The origin and terminus of replication are located opposite to each other in the chromosome and are discerned with high signal to noise ratios by maximal oligonucleotide usage biases on the leading and lagging strand. Genomic DNA structure is rather uniform throughout the chromosome with respect to intrinsic curvature, position preference or base stacking energy. The orthologs and paralogs of A. borkumensis genes with the highest sequence homology were found in most cases among g-Proteobacteria, with Acinetobacter and P. aeruginosa as closest relatives. A. borkumensis shares a similar oligonucleotide usage and promoter structure with the Pseudomonadales. A comparatively low number of only 18 genome islands with atypical oligonucleotide usage was detected in the $A$. borkumensis chromosome. The gene clusters that confer the assimilation of aliphatic hydrocarbons, are localized in two genome islands which were probably acquired from an ancestor of the Yersinia lineage, whereas the alk genes of Pseudomonas putida still exhibit the typical Alcanivorax oligonucleotide signature indicating a complex evolution of this major hydrocarbonoclastic trait.

\section{Introduction}

Alcanivorax borkumensis strain SK2 is a cosmopolitan oil-degrading oligotrophic marine g-proteobacterium (Yakimov et al., 1998). The SK2 strain is the paradigm for hydrocaronoc astic bacteria that are specialized for hydrocarbon degradation but have an otherwise highly restricted substrate spectrum, being capable of utilizing only a few organic acids such as pyruvate, but not simple sugars, for growth (Yakimov et al., 1998; Sabirova et al., 2006). A. borkumensis is present in low abundance in unpolluted environments, but it rapidly becomes the dominant bacterium in oilpolluted open ocean and coastal waters, where it can constitute $80-90 \%$ of the oildegrading microbial community (Harayama et al., 1999; Kasai et al., 2001; 2002; Syutsubo et al., 2001; Röling et al., 2002; Hara et al., 2003; McKew et al., 2007a,b). 
The genome of $A$. borkumensis was recently sequenced and annotated (Schneiker et al., 2006). In this paper, we perform a genome wide comparative genomics analysis and a detailed characterization of the global features of the A. borkumensis strain SK2 genome. This work on A. borkumensis strain SK2 aimed to visualize the prospective potential of genome linguistic approaches for functional and comparative analysis of bacterial genomes.

\section{Results and discussion}

\section{DNA structure and highly expressed genes}

The genome atlas (Fig. 1) shows a combination of some general informative properties of the chromosome. These are structural features (intrinsic curvature, stacking energy and position preference), repeat properties (global direct and inverted repeats) and the main base omposition features (GC skew and percent AT). Stacking energy measures helix rigidity and position preference is a flexibility measure (Jensen et al., 1999; Pedersen et al., 2000). Regions that exhibit low position preference correlate with an enrichment of highly expressed genes (Dlakic et al., 2004; Willenbrock and Ussery, 2007). Examples in A. borkumensis are the rrn operons, the genes encoding ribosomal proteins and the gene cluster labelled rpoC on the atlas which among others encodes RNA polymerase subunits.
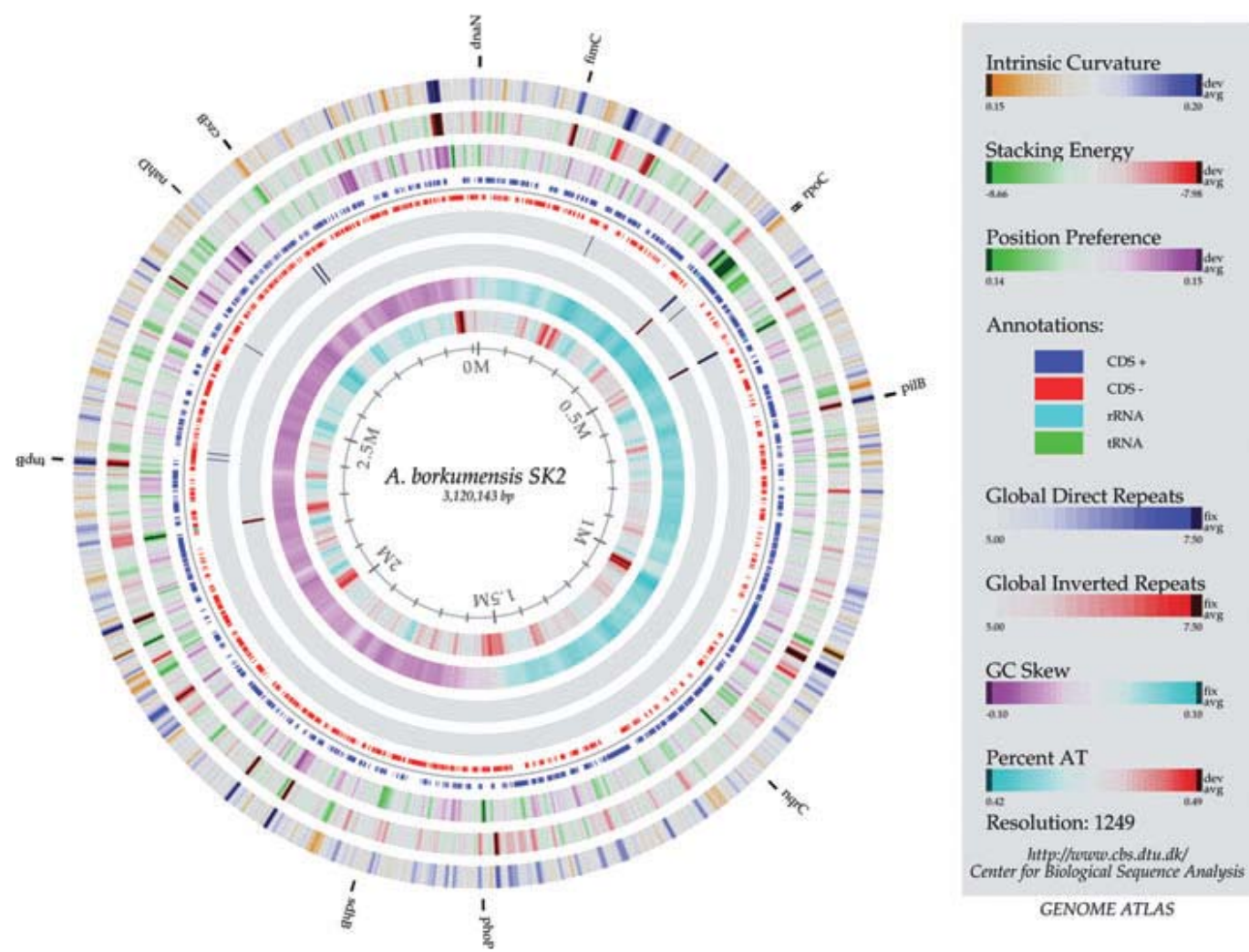

Fig. 1. Genome Atlas of $A$. borkumensis SK2 showing different structural parameters and the distribution of global repeats, GC skew and A $+\mathrm{T}$ contents. Colour intensity increases with the deviation from the average. Values close to the average are shaded 
very light grey; values with more than 3 standard deviations from the average are most strongly coloured.

Low position preference was found to correlate with high codon adaptation indices as the common measure for highly expressed genes (Willenbrock et al., 2006) indicating that the local DNA structure is an important determinant of codon usage and gene expression. Moreover, intrinsic curvature is often encountered upstream of highly expressed genes (Skovgaard et al., 2002) which correlates well with the fact that promoter DNA tends to be more curved than DNA in coding regions (Pedersen et al., 2000). The chromosome is rather homogeneous in all analysed structural features. The number of repeats is low, and the terminus of replication is opposite to the origin of replication as indicated by GC skew (Ussery et al., 2002).

The three rRNA operons organized in the order 16S-23S-5S are located in three areas with low position preference (green marks in the 3 rd circle) and possible upstream regions with high intrinsic curvature (blue in the 1 st circle) near $0.4 \mathrm{Mb}-0.5 \mathrm{Mbases}$ (two regions) and 2.25 Mbases (one region).

\section{Phylogenomics by sequence homology}

The genome of $A$. borkumensis was compared with existing sequence information in other Proteobacteria by constructing phylogenetic trees for each amino acid sequence and organisms for which a similar gene existed. By extracting the phylogenomic information of the resulting 1919 phylogenetic trees a phylome atlas could be constructed (Fig. 2). In most ases the orthologs and paralogs with the highest sequence homology were found among g-Proteobacteria. A substantial proportion of A. borkumensis genes had their closest homolog ues in a- and b-Proteobacteria, but no closest homologue was detected in d- and e-Proteobacteria. Inspection of the collected phylogenetic connections revealed that the most closely related organisms are Acinetobacter sp. and Pseudomonas aeruginosa, although in trees where both Pseudomonas and Acinetobacter are present, A. borkumensis tends to cluster more often with the latter one. No obvious horizontal gene transfers seem to have taken place. Regions around 350.000 and 450.000 are very 'pure' g-proteobacteria regions.

\section{Genome analysis of oligonucleotide usage}

Oligonucleotide usage (OU) has been shown to be a genome specific signature (Pride et al., 2003; Reva and Comparative genomics of Alcanivorax borkumensis 615 

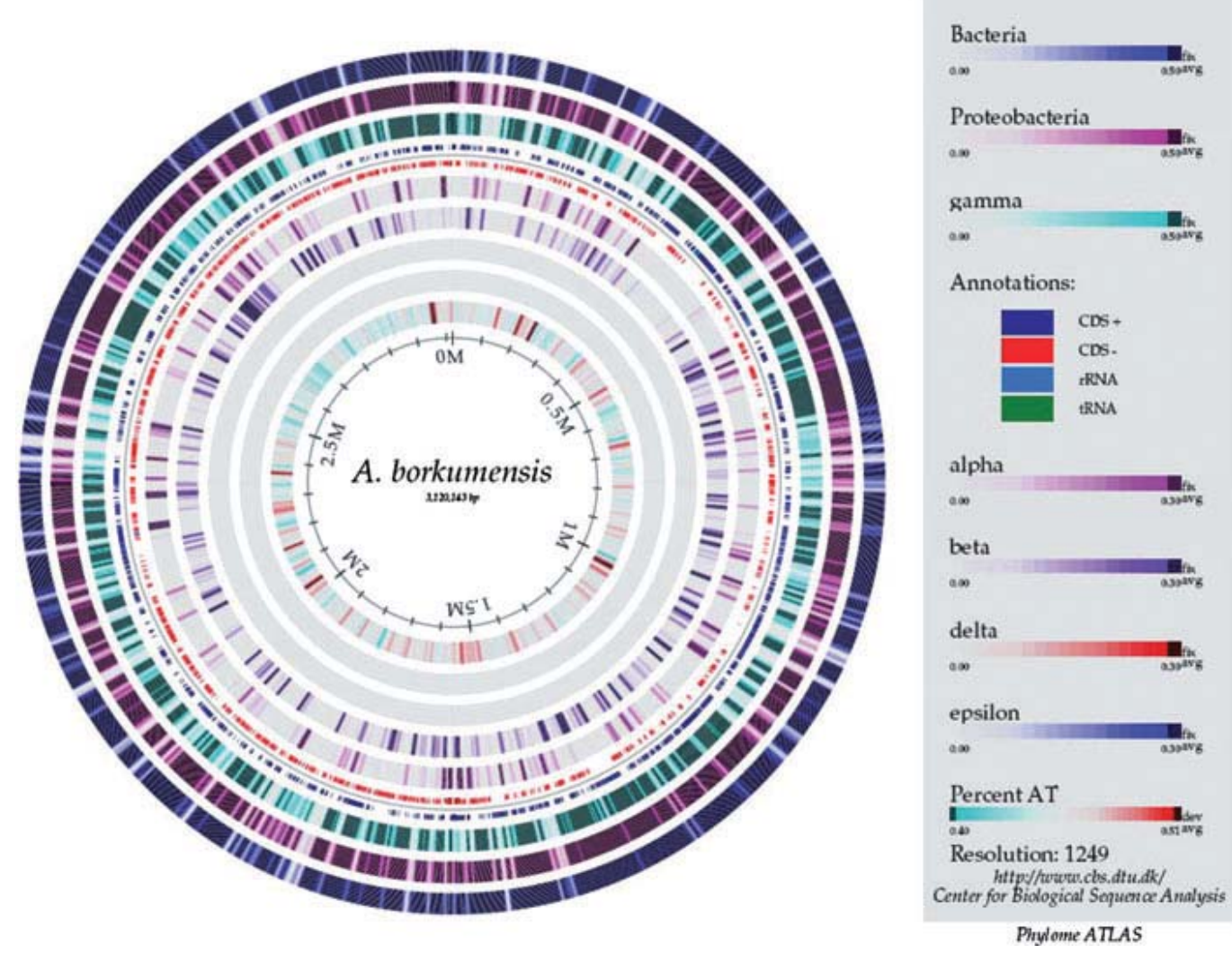

Fig. 2. Phylome Atlas of A. borkumensis SK2 genes indicating their closest bacterial homologues. Each of the concentric circles represents a taxonomic group as described in the figure legend on the right, with the outermost circle corresponding to the topmost feature, and the innermost circle corresponding to the bottom-most feature. Light bands indicate $A$. borkumensis SK2 genes with no homologue in the respective taxonomic group.

Tümmler, 2004). Genomic regions termed the 'core sequences' are characterized by OU patterns being similar to the global pattern of the chromosome. However, many loci with alternative OU patterns typically contribute to in total more than $10 \%$ of a bacterial genome. These loci with atypical OU patterns comprise heterogeneous subsets of parasitic and recent foreign DNA, ancient genes for ribosomal constituents (RNAs and proteins), multidomain genes and non-coding sequences with multiple tandem repeats (Reva and Tümmler, 2005). Hence laterally transferred gene islands can be reliably identified in complete genomes by their atypical oligonucleotide usage (Reva and Tümmler, 2005; Chen et al., 2007; Klockgether et al., 2007). Here, we focused on tetranucleotide usage (TU) parameters because the 256 different tetranucleotide words are optimal to differentiate bacterial genome sequences by the frequency and informativeness of the individual element. TU patterns represent the deviations of tetranucleotide word counts in a given sequence from an equiprobable distribution. Selection and counterselection of the oligonucleotide words are driven by their stereochemical properties such as base stacking energy, propeller twist angle, protein deformability, bendability and position preference (Reva and Tümmler, 
2004). By permutation analysis, the 256 tetranucleotides were assigned to 39 equivalence classes each of which characterized by the same values for the five properties mentioned above (Baldi and Baisnee, 2000). Words of the same equivalence class tend to occur at similar frequencies in a nucleotide sequence (Reva and Tümmler, 2004). Oligonucleotide usage conservation reflects to some extent the phylogeny of microorganisms (Pride et al., 2003; Teeling et al., 2004).

\section{Phylogenomics by tetranucleotide usage analysis}

TU patterns were calculated for all sequenced genomes of g-Proteobacteria. Four examples of TU patterns determined for A. borkumensis SK2, Pseudomonas putida T2440, Escherichia coli K-12 and Shewanella oneidensis MR-1 are shown in Fig. 3. Tetranucleotide words were grouped by the equivalence classes and sorted in order of decrease of the base stacking energy. Figure 4 visualizes the phylogenetic relationships differentiated by TU patterns of $29 \mathrm{~g}$-Proteobacterial taxa each of which represented by not more than a single sequenced strain. A. borkumensis forms a cluster with Pseudomonas, Methylococcus, Xanthomonas and Xylella (Fig. 4). Despite the variation in GC-content, from 52 to $54 \%$ in Xylella and Alcanivorax to more than $65 \%$ in Xanthomonas and Pseudomonas, the TU patterns of these microorganisms are similar and separated from other g-Proteobacteria. There is an abundance of GC-rich tetranucleotides with high base stacking energy in the sequence of $A$. borkumensis SK2 (words belonging to equivalence classes 37-39, 30 and 27) that is similar to the TU pattern of $P$. putida KT2440 (Fig. 3). Words of the AT-rich classes 7, 10, 13 and 32 are significantly underrepresented in both species. The major difference between TU patterns is the abundance of poly A and poly T stretches (words of class 1) in $A$. borkumensis in correspondence with its lower GC-content of 54.7\%. Although E. coli and $S$. oneidensis share a similar GC contents with $A$. borkumensis, their tetranucleotides usage is different from Alcanivorax. The parity of GC with AT in the genome correlates with a balanced use of GC-rich and AT-rich words with high and low base stacking energy. In contrast, words with intermediate values of the base stacking energy (classes 25, 31, 36 and 29) are mostly underrepresented (Fig. 3). The data suggests that oligonucleotide usage drives GC-content and not vice versa. To give another example: the GC-rich words of class 21 are rare in all g-Proteobacteria irrespectively of their GC-content (Fig. 3), but these words are overrepresented in aProteobacteria (Agrobacterium, Bordetella, Caulobacter, Rhizobium). 


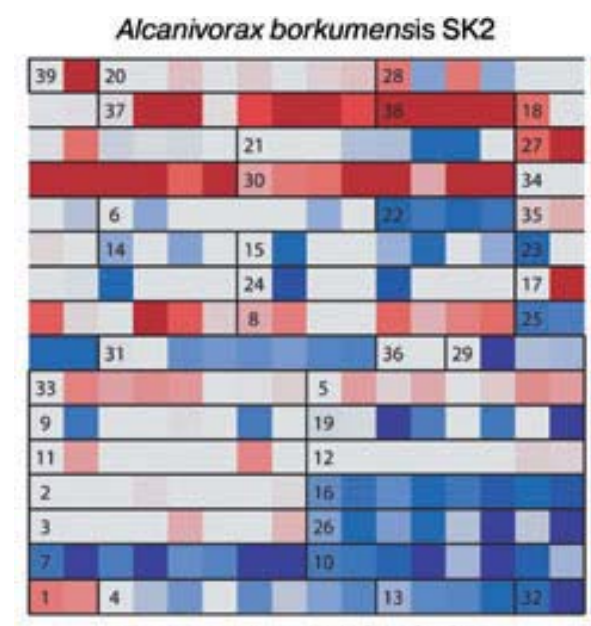

Escherichia coli K12 MG1655

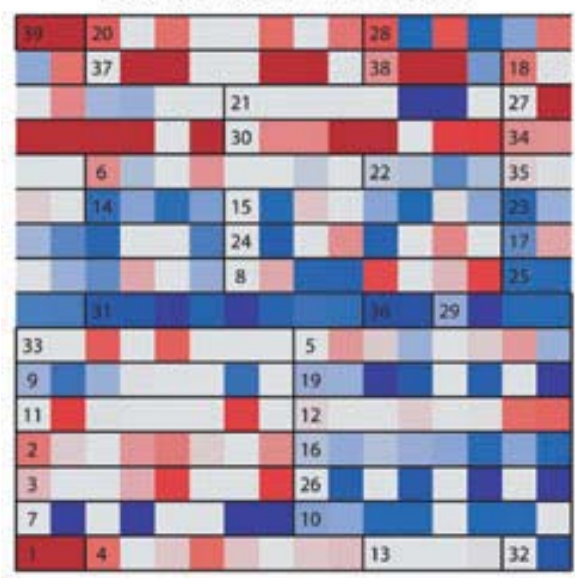

Pseudomonas putida KT2440

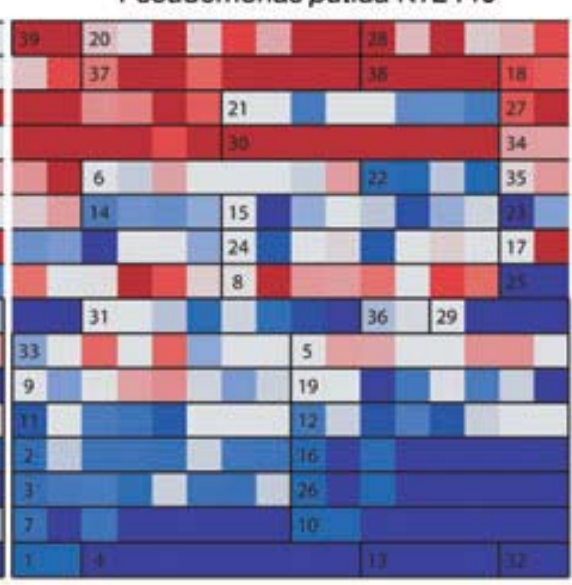

Shewanella oneidensis MR-1

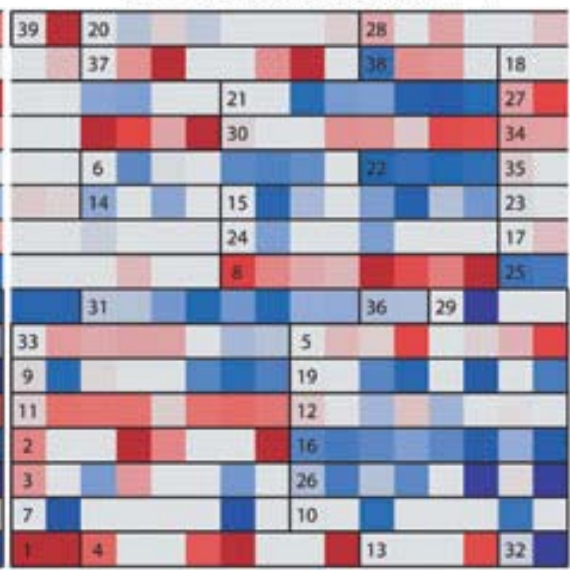

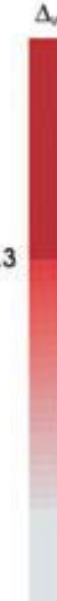

0

$-0.3$

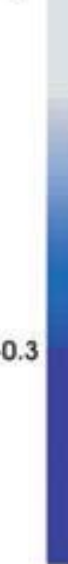

\begin{tabular}{|c|c|c|c|c|c|c|c|c|c|c|c|c|c|c|c|}
\hline 0606 & $\operatorname{GCGC}$ & $A C G C$ & CACG & CGCA & CGTG & GCAC & GCGT & GTGC & TGCG & $A G C B$ & CGAG & СGCT & CTCG & GAGC & GCGA \\
\hline छtc & TCGC & $\operatorname{coc} \theta$ & $\operatorname{ccsc}$ & $\operatorname{coc} c$ & $\cos \theta$ & $G C C C$ & GCGG & GGCO & $G G B C$ & $\cos \theta$ & $\cos C$ & $G C C G$ & $G G C C$ & $A C D B$ & $\operatorname{Cc} A$ \\
\hline CGAC & CGGT & GACC & GGTC & GTळG & TCGG & ACGG & COGT & CGGA & CGTC & GACG & GGAC & GTCC & TCCG & $A G C C$ & CAGC \\
\hline$\triangle C A G$ & CTQG & GCCA & GCTG & GCCT & $T \in O C$ & $A G G C$ & CAGG & СCTG & CTGC & QCAG & GCCT & GGCA & $T \in O C$ & $A T C B$ & CGAT \\
\hline GATC & TCGA & $A M C G$ & ACGA & CGAA & CGIT & GAAC & GTC & TCGT & $\pi C G$ & ACGT & CGTA & GTAC & TACG & ATGC & CATG \\
\hline GCAT & TSCA & $\mathrm{ACAC}$ & CaCA & GTGT & TGTG & ACAG & $A G A C$ & CAGA & CTGT & GACA & GTCT & TCTG & TGTC & ACTC & AGTG \\
\hline CACT & CTCA & GAGT & GTQA & TCAC & TOAG & ACTG & AGTC & CAGT & CTGA & GACT & GTCA & TCAG & TGAC & $A C O C$ & $\mathrm{CACC}$ \\
\hline$\triangle C A C$ & $C O C A$ & GGGT & GGTG & GTGG & TGGG & $A A G C$ & AGCA & CAAG & CTTG & QCAA & GCTT & TGCT & $\pi G C$ & AGAG & СTCT \\
\hline GAGA & TCTC & $A G G B$ & СССт & cCtc & стळ & GAGO & GGAG & GGGA & TCCC & $\operatorname{coc} c$ & $G G G O$ & AGCT & CTAG & GCTA & TAGC \\
\hline ATCC & ATGG & CATC & CCAT & GATG & GGAT & TCCA & TGGA & $\triangle A C C$ & $A C C A$ & CAAC & CCAA & GGIT & GITG & TGGT & TIGG \\
\hline$A A G G$ & $A G G A$ & $\mathrm{CCTT}$ & crTC & GAAG & GGA & TCCT & $\pi C C$ & ACCT & AGGT & ССТА & CTAC & GGTA & GTAG & TACC & TAGG \\
\hline AATC & ATCA & ATTG & CAAT & GAIT & TCAA & TGAT & TTGA & AATG & ATGA & ATTC & CATT & GAAT & TCAT & TGAA & TTCA \\
\hline AAAC & AACA & ACAA & CANA & GII & TGT & ताG & $\pi \mathrm{TH}$ & ACAT & ATAC & ATGT & CATA & GTAT & TACA & TATG & TGTA \\
\hline$\triangle A A B$ & AMGA & AGAA & $\mathrm{cm}$ & GAAA & $\mathrm{TCT}$ & חाст & $\pi \mathrm{T}$ & AGAT & ATAG & ATCT & CTAT & GATA & THGA & TATC & TCTA \\
\hline AACT & ACTA & AGT & CTAA & GTRA & TAAC & TAGT & TTAG & AAGT & ACTT & AGTA & CTIA & GTAA & TAAG & TACT & TTAC \\
\hline$\triangle A A A$ & $\pi \pi$ & AMAT & AATA & ATAA & AITT & TAAA & TATT & $\pi A T$ & $\pi T A$ & AATT & ATTA & TAAT & TIAA & ATAT & TATA \\
\hline
\end{tabular}

Fig. 3. Tetranucleotide usage patterns of $A$. borkumensis SK2, P. putida KT2440, E. coli K12 MG1655 and S. oneidensis MR-1. The deviation Dw of observed from expected counts is shown for all 256 tetranucleotide words ( $16 ¥ 16$ cells) by colour 
code (right bar). Tetranucleotides are grouped into 39 classes of equivalent structural features (Baldi and Baisnee, 2000) and sorted by decreasing base stacking energy row-by-row starting at the upper left corner (class 39). The words corresponding to the cells in colour plots are shown in the table in lower part of the figure.

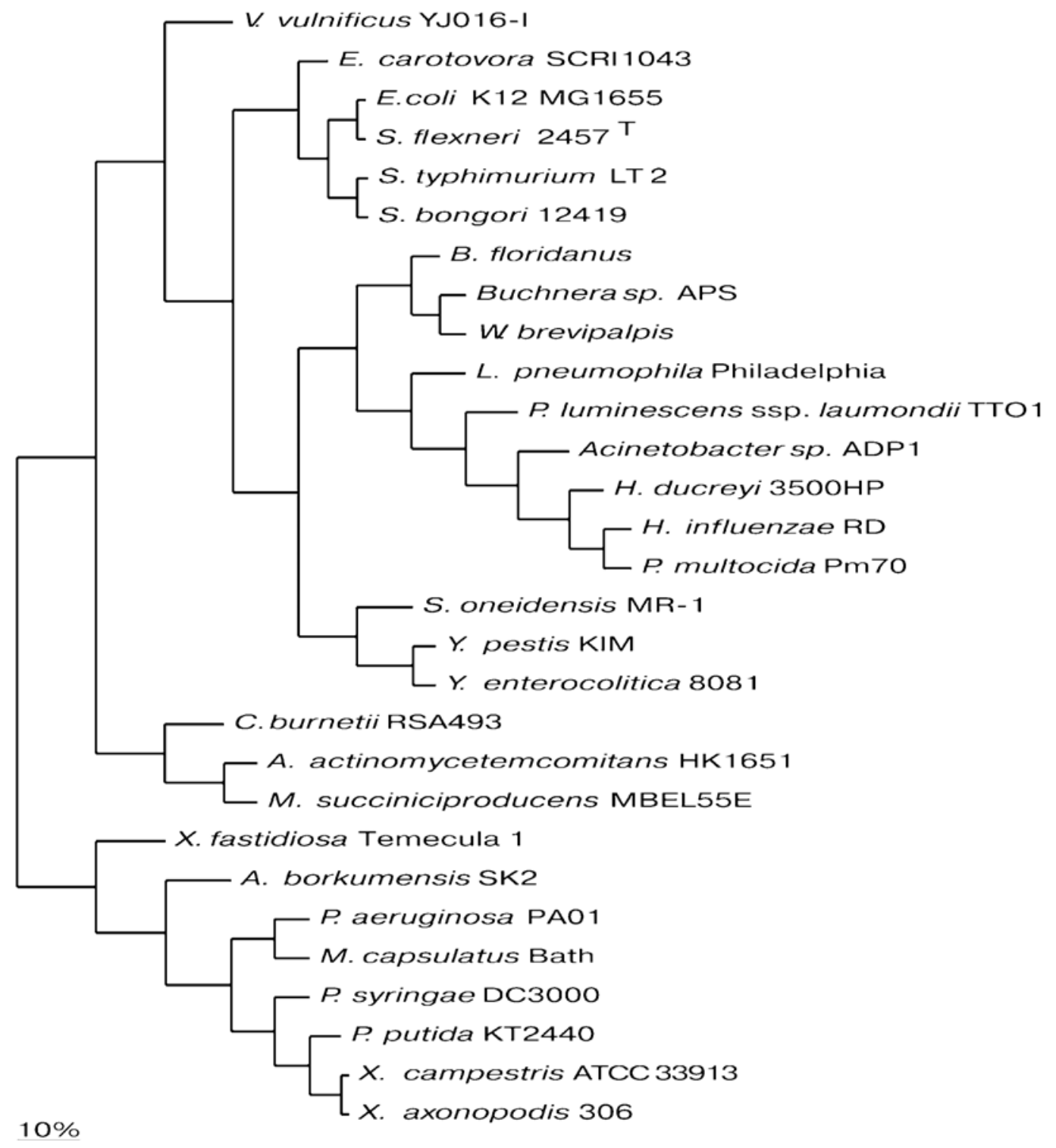

Fig. 4. Tree of the similarity of TU patterns of completely sequenced g-roteobacteria strains. Distance $D$-values (see Experimental procedures) between two TU patterns were calculated, and the tree was constructed from the distance matrix of all $D$-values by the minimum evolution neighbour-joining method (Saitou and Nei, 1987). 


\section{Anomalous local TU patterns in the A. borkumensis genome}

A. borkumensis shares a common taxonomic group with Pseudomonas, Methylococcus, Xanthomonas and Xylella. Although the TU patterns are genome specific signatures, the oligonucleotide usage may vary locally in segments made up by horizontally acquired elements, phylogenetically ancient genes such as rRNAs or genes with peculiar codon usage (Reva and Tümmler, 2004; 2005). In other words, anomalous local TU patterns can be expected for the most recent and the most ancient genes. Local TU patterns were calculated in $8 \mathrm{kbp}$ long overlapping sliding windows in steps of $2 \mathrm{kbp}$. Distances D between local and global TU patterns are shown in Fig. 5. The 18 regions with $D$-values above the $95 \%$ confidence interval are listed in Table 1. Three clusters with anomalous $D$-values encode ribosomal RNAs that belong to the most ancient and conserved elements of all bacterial genomes. All the other 15 regions with atypical TU most likely were recently acquired, three of which contain transposase genes. In total 11 transposases were annotated in the A. borkumensis SK2 genome but for five of them no significant deviations of the local TU atterns were detected in adjacent regions. If inserted mobile elements had lost their mobility due to disruptive mutations, they undergo an amelioration process smoothing the differences in oligonucleotide usage between inserts and the host genome and thus cannot be detected by anomalous TU patterns anymore (Pride et al., 2003). Five regions with high $D$-values (Fig. 5) only encode hypothetical proteins (Table 1). One further region contains genes of the type II secretion system and two regions encode type IV pili biogenesis proteins the latter of which are known to have spread among proteobacteria by horizontal transfer with the original codon usage and GC content being retained (Spangenberg et al., 1997).

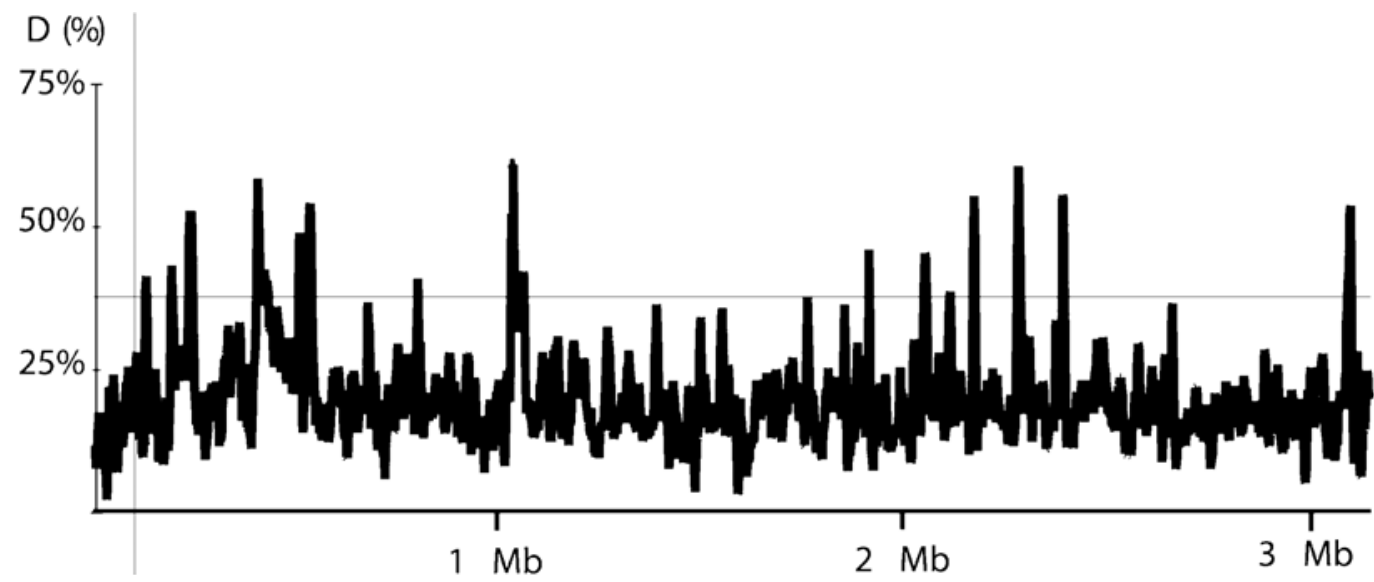

Fig. 5. Deviations of TU patterns in local regions of A. borkumensis SK2 chromosome. Local TU patterns were determined in $8 \mathrm{kbp}$ sliding window in steps of $2 \mathrm{kbp}$. D, the distance betweeen local and chromosomal tetranucleotide patterns as defined in Experimental procedures, is plotted versus the coordinates of the chromosome starting from the putative replication origin. The upper border of the $95 \%$ confidence interval of $D$-values is shown by the horizontal line.

The most extended region with high $D$-values encodes a cluster of genes for glycosyltransferases and polysaccharide biosynthesis proteins (Abo_858-Abo_880: 1 
$018000-1060000 \mathrm{bp}$ ) characterized by the second largest $D$-value and low GCcontent (minimum 45\% GC). The region terminates abruptly after Abo_880 at an AsntRNA gene. The TU pattern of the locus was compared with those of 177 sequenced bacterial chromosomes, 316 plasmids and 104 phages (Reva and Tümmler, 2004). The pattern was distant from all analysed sequences. The best hit of $D=34.9 \%$ was observed for the 5833 bp large bacteriophage Pf3 that infects $P$. aeruginosa harbouring the RP1 plasmid (Luiten et al., 1985). A stretch of 1550 bp upstream of the tRNA gene is $48 \%$ identical in nucleotide sequence with the Pf3 sequence (2344$4078 \mathrm{bp}$ ). According to this in silico finding we propose that this gene island was captured from a phage that typically target the 3 '-end of a tRNA gene (Dobrindt et al., 2004).

The alkB genes encoding the degradation of alkanes which is the prominent namegiving feature of the taxon Alcanivorax, are located in two islands (Schneiker et al., 2006) with anomalous TU patterns (Table 1). Very close homologues were identified in marine bacteria and Pseudomonas species (Schneiker et al., 2006). The alkane hydroxylase gene cluster is widely distributed among hydrocarbon-utilizing gProteobacteria due to its possible horizontal transfer (van Beilen et al., 2001; 2004).

Table 1. Chromosomal regions of $A$. borkumensis with atypical TU patterns.

\begin{tabular}{|c|c|c|c|}
\hline \multicolumn{2}{|c|}{ Coordinates } & \multirow[b]{2}{*}{$D^{a}(\%)$} & \multirow[b]{2}{*}{ Annotation } \\
\hline Left & Right & & \\
\hline 126000 & 140000 & 42.20 & $\begin{array}{l}\text { Abo_114-120: lys } R \text { transcriptional regulator, haloacid dehalogenase hydrolase, amiC amidase, gntR } \\
\text { transcriptional regulator, alkB2 alkane monooxygenase, type I pili biogenesis proteins }\end{array}$ \\
\hline 190000 & 198000 & 40.47 & $\begin{array}{l}\text { Abo_172-178: ilvD-1 dihydroxy-acid dehydratase, conserved hypothetical proteins, } \\
\text { long-chain-fatty-acid-CoA ligase, acyl-CoA dehydrogenases }\end{array}$ \\
\hline $\begin{array}{l}234000 \\
400000\end{array}$ & $\begin{array}{l}245000 \\
408000\end{array}$ & $\begin{array}{l}47.95 \\
49.42\end{array}$ & $\begin{array}{l}\text { Abo_209-214: conserved hypothetical proteins, transposase, type II secretion system proteins } \\
\text { first_operon for rRNAs }\end{array}$ \\
\hline 502000 & 510000 & 46.26 & $\begin{array}{l}\text { Abo_439-446: ispA lipoprotein signal peptidase, } f \mathrm{kpB} \text { peptidyl-prolyl cis-trans isomerase, ispH } \\
\text { hydroxymethylbutenyl pyrophosphate reductase, type IV pili biogenesis proteins, conserved } \\
\text { hypothetical proteins }\end{array}$ \\
\hline 526000 & 534000 & 43.41 & second operon for rRNAs \\
\hline 670000 & 678000 & 40.29 & Abo_581-583: type IV pili biogenesis proteins \\
\hline 792000 & 800000 & 43.00 & Abo_2680-2681: hypothetical proteins \\
\hline 1020000 & 1056000 & 50.43 & Abo_859-878: polysaccharide biosynthesis proteins \\
\hline 1742000 & 1750000 & 40.88 & Abo_1439: periplasmic binding domain/transglycosylase SLTdomain fusion \\
\hline 1892000 & 1900000 & 46.32 & Abo_2841-2847: hypothetical proteins \\
\hline 2026000 & 2034000 & 41.90 & $\begin{array}{l}\text { Abo_1668-1671: conserved hypothetical proteins, } 3 \text { transposases, siderophore biosynthesis protein, } \\
\text { glycosyl transferase }\end{array}$ \\
\hline 2088000 & 2096000 & 40.65 & Abo_ 1707-1708: conserved hypothetical proteins \\
\hline 2146000 & 2154000 & 47.05 & $\begin{array}{l}\text { Abo_2897-2905: iscA iron-binding protein IscA, metal-sulfur cluster biosynthetic enzyme, sufE Fe-S } \\
\text { metabolism associated domain protein, iscS cysteine desulfurase, } r \text { t2 family protein, hypothetical } \\
\text { proteins, SIR2-like transcriptional silencer }\end{array}$ \\
\hline 2254000 & 2262000 & 49.71 & third operon for rRNAs \\
\hline 2364000 & 2372000 & 52.56 & Abo_1942: penicillin-binding protein, hypothetical proteins, 2 transposases \\
\hline 2632000 & 2640000 & 40.17 & Abo_2979-2984: hypothetical proteins \\
\hline 3060000 & 3076000 & 42.94 & $\begin{array}{l}\text { Abo_2516-3066: } \mathrm{Na}+/ \mathrm{H}+\text { antiporter, alkS alkB1GHJ regulator, alkB1 alkane monooxygenase, } \\
\text { alkG rubredoxin, aldH aldehyde dehydrogenase, hypothetical proteins }\end{array}$ \\
\hline
\end{tabular}

a. D, distance between local and chromosomal TU patterns as defined in Experimental procedures.

The role of these genes in the degradation of short-chain $n$-alkanes by A. borkumensis SK2 and AP1 was experimentally proven (Smits et al., 2002; Hara et al., 2004; Sabirova et al., 2006). Interestingly, the two regions comprising of alkS, alkB1, alkG and ald $H$ alkanedegradation genes and of alkB2 and transcriptional regulators, respectively (Table 1), are as similar to each other in their TU patterns $(\mathrm{D}=34.3 \%)$ as each of them is to Yersinia pestis $(\mathrm{D}=32.2 \%$ for alkB1, $\mathrm{D}=33.4 \%$ for alkB2), Yersinia enterocolitica $(\mathrm{D}=29.5 \%$ for alkB1, $\mathrm{D}=34.4 \%$ for alkB2) and Shewanella 
neidensis MR-1 $(\mathrm{D}=32.5 \%$ for alkB1, $\mathrm{D}=42.4 \%$ for alkB2). This data suggests that the alkB1 and alkB2 genes were delivered to A. borkumensis from an ancestor of the Yersinia lineage. The AlkB1 amino acid sequences of $A$. borkumensis strains AP1 and SK2 are highly homologous to that of P. putida strains P1 and GPO1 (van Beilen et al., 2001; 2004; Smits et al., 2002; Hara et al., 2004), but their TU patterns are not that similar $(\mathrm{D}=37.1)$. Surprisingly, the TU pattern of the alkB cluster of $P$. putida is significantly more similar with the global TU pattern of the whole A. borkumensis chromosome (16.7\%, strain GPO1, 19\%, strain P1), but more distant from the $P$. putida KT2440 chromosome (30.1\% and $30.3 \%)$ D-values of 17 or $19 \%$ are within the first quartile (0-26\%) far below the median value of $28.4 \%$ for local TU patterns of the A. borkumensis chromosome (Fig. 5) indicating that. the P. putida alkB gene behaves as if it were part of the Alcanivorax core genome. We note the striking phenomenon that there was converging evolution of the coding sequence of the catabolic alk transposon in Alkanivorax and Pseudomonas, but that the genes retained the oligonucleotide signature of their donors, most likely Alkanivorax for Pseudomonas and Yersinialike organisms for Alkanivorax.

\section{Origin of replication}

The GC skew plotted in the seventh circle of the genome atlas (Fig. 1) reflects a general bias of purines towards the leading strand of DNA replication, however, it has almost no orrelation the structural properties of DNA (Skovgaard et al., 2002). The GC skew is often useful when locating the origin and terminus of replication (Jensen et al., 1999). The circle is blue on the right side and purple on the left side. The two big gaps of colours in the top and in the bottom of the circle may be the origin and the terminus of replication. This may also be visualized more clearly in the origin plot (Fig. 6) (Worning et al., 2006). Here, the difference between hypothetical leading and lagging strand is plotted (red) for various positions on the chromosome. The peaks indicating maximal oligonucleotide skew correspond to origin and terminus. The terminus was identified as the peaks showing low $\mathrm{G} / \mathrm{C}$ weighted strand bias at $1502000 \mathrm{bp}$ position. The origin was identified as the other peak at 3118000 bp position. The signal to noise of 14.0 was among the top $10 \%$ of sequenced Proteobacteria, indicating a big difference between leading and lagging strand making the prediction of origin very confident.

\section{Structural analysis of promoter regions}

Structural features of the genomic DNA may indicate promoter regions, as promoters normally have high curvature, melt easily and are more rigid. The DNA structural parameters mentioned earlier (position preference, stacking energy, and intrinsic curvature) together with AT content and DNAse sensitivity (Brukner et al., 1995) were compiled into a structural profile of all upstream regions of $A$. borkumensis (see section Experimental procedures). The profile uses z-scores to measure how the average value of the properties vary from minus $400 \mathrm{bp}$ to $400 \mathrm{bp}$ around the translation start (Fig. 7). A. borkumensis has only a coding density of $87 \%$ causing a wider spacer of the intergenic region and this appears to give rise to a larger and wider peak of curvature, stacking energy and AT content (Fig. 7A). For comparison we also analysed the promoter profile of another ocean bacterium, Candidatus Pelagibacter ubique HTCC1062 (Giovannoni et al., 2005), an example of a highly streamlined genome with a coding density of $96 \%$. Here we observed a much weaker curvature signal, and the distribution of stacking energy and AT content was more narrow and had higher maxima (Fig. 7B). 


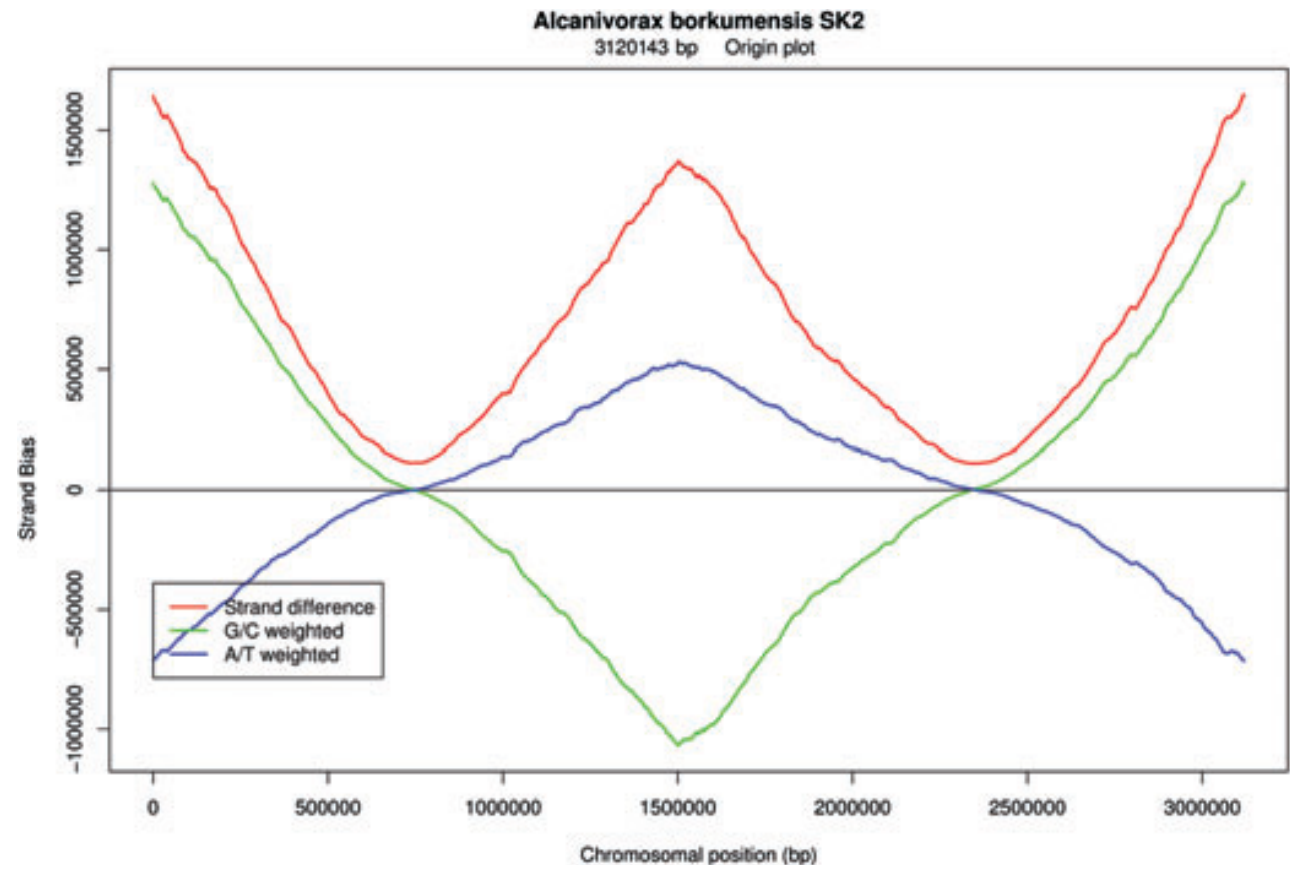

Fig. 6. Localization of the origin and the terminus of replication in the A. borkumensis SK2 chromosome derived from strand bias curves: the median oligonucleotide skew curve (red), the GC weighted median (green) and the AT weighted median (blue) (Worning et al., 2006).

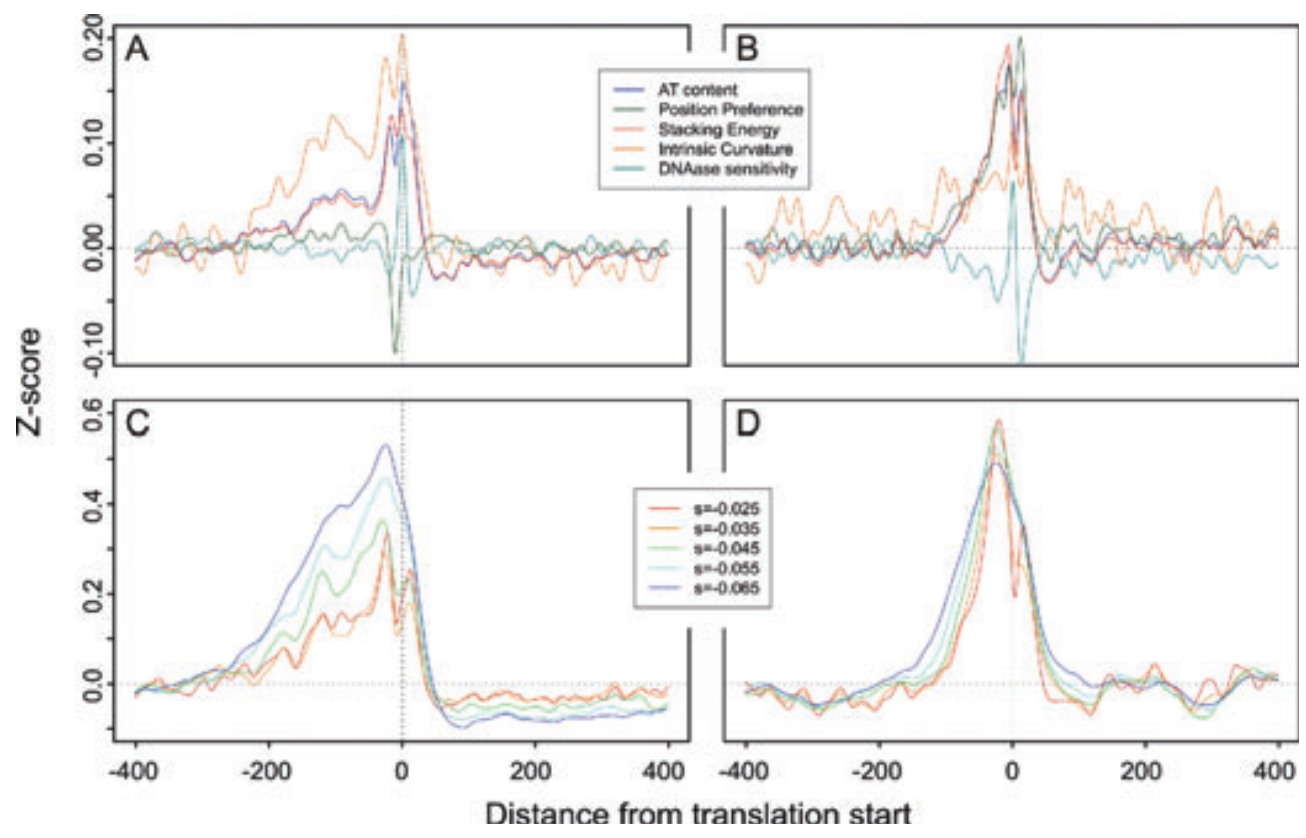

Fig. 7. Profile of structural properties of promoter regions (A and B) and probabilities of opening during stress-induced DNA duplex destabilization at various super-helical densities $(\mathrm{C}$ and $\mathrm{D}$ ) in the $\mathrm{A}$. borkumensis SK2 (A and $\mathrm{C}$ ) and Candidatus Pelagibacter ubique HTCC1062 (B and D) chromosomes. Each annotated gene was 
aligned at the translation start site and the average values for the SIDD probabilities, AT-content, position preference, stacking energy, intrinsic curvature and DNase sensitivity were calculated at each position in the alignment. The values were subsequently converted into z-scores, using the average and standard deviation of the entire chromosome. Values are smoothed over a $5 \mathrm{bp}$ window.

Next, the probability of opening during stress-induced DNA duplex destabilization was computed by using the program SIDD (Wang et al., 2004), covering five different values of the super-helical density $\mathrm{s}=\{-0.025,-0.035,-0.045,-0.055,-$ $0.065\}$. As super-coiling is being pushed, the probability of opening increases at lower super-helical densities in A. borkumensis (Fig. 7C). In contrast, a narrower SIDD profile that exhibits only minor dependence on super-helical density (Fig. 7D), was calculated for the Candidatus Pelagibacter ubique HTCC1062 genome.

The structural profile for the promoter regions of $A$. borkumensis was compared with that of closely related species as found above (see Fig. 4). Generally, it looked more like the promoter profile of members of the Pseudomonadales than the general comparison organism, E. coli. Moreover, the promoter profile was very different compared with the promoter profile of $X$. fastidiosa strains, even though they where very similar with regard to their TU profile (see Fig. 4). The promoter profiles for the above mentioned organisms may be found at our website http://www.cbs.dtu.dk/services/GenomeAtlas/).

\section{Amino acid and codon usage}

We have examined the codon and amino acid usage of $A$. borkumensis and compared this with both the usage of bacteria in general and of 16 oceanic bacteria (Entrez project IDs 230, 10 645, 12 530, 13 233, 13 239, 13 282, 13 642, 13 643, 13 654, 13 655, 13 902, 13 906, 13 910, 13 911, 13 989, 15 660) Willenbrock et al., 2006). In

Fig. 8, the codon usage plot of $A$. borkumensis is superimposed on the cumulative plot of all completely sequenced bacteria in public databases $(N=518$, Fig. $8 \mathrm{~A})$ or of that of 16 oceanic bacteria (Fig. 8B). A few codons are differentially utilized in $A$. borkumensis (GUC, CUG), but all values are within the range of three standard deviations. In other words, codon usage of $A$. borkumensis resides within the typical range of eubacteria.

Interestingly, the sequenced oceanic bacteria share a very similar amino acid usage (Fig. 8D), whereas broad variations thereof were noted amongst all sequenced bacteria that represent the whole spectrum of habitats (Fig. 8C). A. borkumensis roughly follows the profile of the oceanic bacteria, although cysteine, tryptophan, leucine, proline, arginine, serine are under-utilized, and glutamic acid, lysine, phenylalanine, histidine, methionine, and tyrosine are over-utilized - all exceeding the threestandard deviation boundaries. 


\section{Conclusion}

Inspection of the collected phylogenetic connections revealed that the most closely related organisms are Acinetobacter sp. and Pseudomonas aeruginosa, although in trees where both Pseudomonas and Acinetobacter are present, A. borkumensis tends to cluster more often with the latter one.
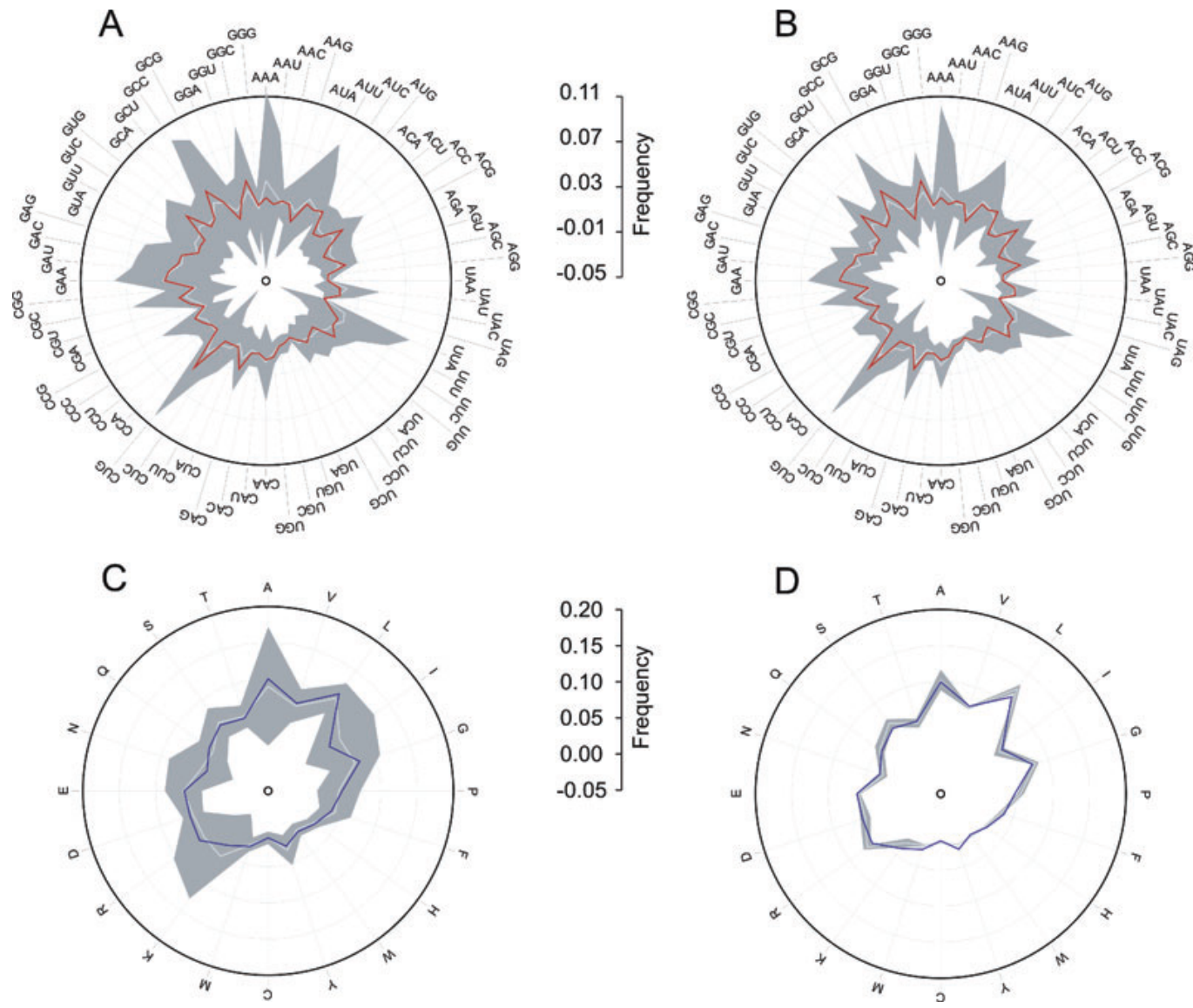

Fig. 8. Codon usage (A and $\mathrm{B}$ ) and amino acid usage (C and D) of $A$. borkumensis SK2 compared with those of 518 completely sequenced bacteria (A and C) or compared with those of 16 sequenced oceanic bacteria. Frequencies of amino acids and codons were counted for each genome and normalized. Mean value (grey line) and three standard deviations (grey solid area) represent the global usage of individual codons (A and $\mathrm{B}$ ) and amino acids $(\mathrm{C}$ and $\mathrm{D})$ in the 518 (A and $\mathrm{C}$ ) or 16 (B and $\mathrm{D})$ reference genomes. The red line (A and $\mathrm{B}$ ) shows the codon usage and the blue line $(\mathrm{C}$ and $\mathrm{D})$ shows the amino acid usage of $A$. borkumensis.

The major structural feature of the $A$. borkumensis chromosome is its symmetry and homogeneity. The genome contains only very few regions with extraordinarily low or high curvature, position preference or base stacking energy. The chromosomal frame is symmetric: The origin and the terminus of replication are located opposite to each 
other in the chromosome and are clearly discerned by maxima of oligonucleotide usage biases between leading and lagging strand. The genetic repertoire of $A$.

borkumensis is most similar to that of Acinetobacter and $P$. aeruginosa. Moreover, $A$. borkumensis shares a similar oligonucleotide usage with the Xanthomonadales and Pseudomonadales indicating close phylogenetic relationships with these orders in accordance with 16S rDNA sequence relatedness (Schneiker et al., 2006). Amongst this subgroup of completely sequenced genomes, the $A$. borkumensis chromosome harbours the relatively lowest number of genome islands with atypical tetranucleotide usage. $P$. putida KT2440, for example, carries threefold more islands per Megabase in its chromosome (Weinel et al., 2002). Interestingly, one of the three enzyme systems that are upregulated in alkane-grown cells (Sabirova et al., 2006), the well-known alkB1 cluster, is encoded by genome islands. The molecular evolution of the alk genes that are encoded by a catabolic transposon (van Beilen et al., 2001) is remarkable: the Alcanivorax genes were probably acquired from the Yersinia lineage, whereas the $P$. putida genes exhibit the typical Alcanivorax tetranucleotide signature. Horizontal gene transfer was relevant to confer the - probably - most important metabolic trait to A. borkumensis, but otherwise the stable seawater habitat apparently did not favour the shuffling and exchange of genes with other taxa. Instead a symmetric and structurally homogeneous chromosome evolved that lacks numerous metabolic traits (Yakimov et al., 1998; Schneiker et al., 2006) found in their versatile Pseudomonas relatives which are endowed with twofold larger chromosomes (Stover et al., 2000; Nelson et al., 2002).

\section{Experimental procedures}

\section{Genomic sequence}

The comparative genomics analyses were based on the genomic sequence of $A$. orkumensis SK2 (Golyshin et al., 2003) and its annotation (Schneiker et al., 2006).

\section{Atlas visualization}

Atlases, developed in house, make it possible to visualize correlations between position dependent information contained within a chromosome. Circular graphical representations of the entire $A$. borkumensis genome were created using the atlas visualization tool, GeneWiz. Each feature, such as AT content is represented by a separate circle in the atlas. Typically, mean values are pictured in grey and extreme values are highlighted in a user defined colour (Pedersen et al., 2000).

Phylome atlas. For each amino acid sequence, hylogenetic trees were automatically constructed as described in Sicheritz-Ponten and Andersson (2001). The phylogenomic information of the resulting 1919 phylogenetic trees was extracted and analysed in the PyPhy system.

Genome atlas. The genome atlas is a combination of some general informative properties. These are some structural features (intrinsic curvature, stacking energy and position preference), some repeat properties (global direct and inverted repeats) and the main base composition features (GC skew and percent AT). Intrinsic curvature was calculated using the CURVATURE software (Shpigelman et al., 1993). Stacking energy of a DNA segment was determined by the method of Ornstein and colleagues 
(1978). Position preference was based on a trinucleotide model that estimates the helix flexibility (Satchwell et al., 1986). Base composition is generally divided into AT content and GC skews. Both were calculated from the nucleotide sequence. Global direct and inverted repeats were found using variations of an algorithm that finds the highest degree of homology for a $15 \mathrm{bp}$ repeat within a window of length 100 bp (Jensen et al., 1999).

\section{Codon and amino acid usage}

Codon and amino acid usage were calculated from all coding regions in the genome as annotated in the GenBank entries. The relative synonymous codon usage was calculated by comparing the codon distribution from a set of highly expressed genes with a background distribution estimated from the codon usage of all coding regions in the genome (Willenbrock et al., 2006). In order to identify a set of constitutively highly expressed genes in $A$. borkumensis, the reference set of 27 very highly expressed Escherichia coli genes originally compiled by Sharp and Li (1986) was aligned at the protein level against all genes annotated in the GenBank entry using BLASTP version 2.2.9 (Altschul et al., 1997). For each of these very highly expressed genes, the gene with the best alignment was added to a set of very highly expressed genes if it had an E-value below 10-6.

\section{TU patterns}

Overlapping tetranucleotide words were counted in the bacterial nucleotide sequences by shifting the window in steps of 1 nucleotide. The total word number in a circular sequence equals to the sequence length. The observed counts of words $(\mathrm{Co})$ were compared with the expected counts of words (Ce). Assuming the same distribution frequency for all words irrespective of their composition and sequence mononucleotide content, $C$ e matches the ratio of the sequence length to the number of different tetranucleotide words $N w$ (256 for tetranucleotides). The deviation Dw of observed from expected counts is given by

$\Delta \mathrm{w}=(\mathrm{Co}-\mathrm{Ce}) \times \mathrm{Co}^{-1}$

For the comparison of sequences by TU patterns, the words in each sequence were ranked by Dw values. Rank numbers instead of word counts were used to simplify pattern comparison and to remove sequence length bias. The distance $D$ between two patterns was calculated as the sum of absolute distances between ranks of identical words in patterns $i$ and $j$ as follows and expressed as a percent of the possible maximal distance:

$$
\begin{aligned}
& \Sigma \text { rank w,i - rank w,j } \\
& \mathrm{W} \\
& D(\%)=100 \times \\
& D \max
\end{aligned}
$$

where

$$
D \max ={ }_{2}^{N w(N w-1)}
$$


Dmax is the maximal distance that is theoretically possible between two patterns. For TU patterns $N w$ is 256 . For more information about methods of oligonucleotide usage statistics see Reva and Tümmler (2004; 2005).

\section{Origin plot}

The origin plot was constructed as described in Worning and colleagues (2006). In brief, the difference between a hypothetical leading and lagging strand is plotted for various positions on the chromosome. The frequencies of all oligonucleotides from 2mers to 8-mers on the leading and lagging strands in a $60 \%$ window are counted and the information content was calculated and summarized over all oligos for every putative origin. The $\mathrm{G} / \mathrm{C}$ and $\mathrm{A} / \mathrm{T}$ weighted strand bias were included to distinguish between origin and terminus.

\section{Structural profile of the promoter region}

Each annotated gene was aligned at the translation start site and the average values for five DNA structural features (AT content, position preference, stacking energy, intrinsic curvature, DNase sensitivity; see chapter on Genome Atlas) were calculated at each position in the alignment. The values was subsequently centered and scaled and smoothed within a 5 bp window using Gaussian smoothing.

\section{References}

Altschul, S.F., Madden, T.L., Schaffer, A.A., Zhang, J., Zhang, Z., Miller, W., and Lipman, D.J. (1997) Gapped BLAST and PSI-BLAST: a new generation of protein database search programs. Nucleic Acids Res 25: 3389- 3402.

Baldi, P., and Baisnee, P.F. (2000) Sequence analysis by additive scales: DNA structure for sequences and repeats of all lengths. Bioinformatics 16: 865-889.

Van Beilen, J.B., Panke, S., Lucchini, S., Franchini, A.G., Rothlisberger, M., and Witholt, B. (2001) Analysis of Pseudomonas putida alkane-degradation gene clusters and flanking insertion sequences: evolution and regulation of the alk genes.

Microbiology 147: 1621-1630.

Van Beilen, J.B., Marin, M.M., Smits, T.H.M., Röthlisberger, M., Franchini, A.G., Witholt, B., and Rojo, F. (2004) Characterization of two alkane hydroxylase genes from the marine hydrocarbonoclastic bacterium Alcanivorax borkumensis. Environ Microbiol 6: 264-273.

Brukner, I., Sanchez, R., Suck, D., and Pongor, S. (1995) Sequence-dependent bending propensity of DNA as revealed by DNase I: parameters for trinucleotides. EMBO J 14: 1812-1818.

Chen, X.-H., Koumoutsi, A., Scholz, R., Eisenreich, A., Schneider, K., Schneider, I., et al. (2007) Comparative analysis of the complete genome sequence of the plant growth promoting Bacillus amyloliquefaciens FZB42. Nat Biotechnol 25: 1007-1014. 
Dlakic, M., Ussery, D., and Brunak, S. (2004) DNA bendability and nucleosome positioning in transcriptional regulation. In DNA Conformation and Transcription. Ohyama, T. (ed.). Austin, TX: Landes Bioscience, pp. 198-211.

Dobrindt, U., Hochhut, B., Hentschel, U., and Hacker, J. (2004) Genomic islands in pathogenic and environmental microorganisms. Nat Rev Microbiol 2: 414-424.

Giovannoni, S.J., Tripp, H.J., Givan, S., odar, M., Vergin, K.L., Baptista, D., et al. (2005) Genome streamlining in a cosmopolitan oceanic bacterium. Science 309: $1242-1245$.

Golyshin, P.N., Martins Dos Santos, V.A., Kaiser, O., Ferrer, M., Sabirova, Y.S., Lunsdorf, H., et al. (2003) Genome sequence completed of Alcanivorax borkumensis, a hydrocarbon-degrading bacterium that plays a global role in oil removal from marine systems. J Biotechnol 106: 215-220.

Hara, A., Syutsubo, K., and Harayama, S. (2003) Alcanivorax which prevails in oilcontaminated seawater exhibits broad substrate specificity for alkane degradation. Environ Microbiol 5: 746-753.

Hara, A., Baik, S.H., Syutsubo, K., Misawa, N., Smits, T.H., van Beilen, J.B., and Harayama, S. (2004) Cloning and functional analysis of alkB genes in Alcanivorax borkumensis SK2. Environ Microbiol 6: 191-197.

Harayama, S., Kishira, H., Kasai, Y., and Shutsubo, K. (1999) Petroteum biodegradation in marine environments. J Mol Microbiol Biotechnol 1: 63-70.

Jensen, L.J., Friis, C., and Ussery, D.W. (1999) Three views of microbial genomes. Res Microbiol 150: 773- 777.

Kasai, Y., Kishira, H., Sasaki, I., Syutsubo, K., Watanabe, K., and Harama, S. (2002) Prodominant growth of Alcanivorax strains in oil-contaminated and nutrientsupplemented sea water. Environ Microbiol 4: 141-147.

Kasai, Y., Kishira, H., Syutsubo, K., and Harayama, S. (2001) Molecular detection of marine bacterial populations on beaches contaminated by the Nakhodka tanker oilaccident. Environ Microbiol 3: 246-255.

Klockgether, J., Würdemann, D., Reva, O., Wiehlmann, L., and Tümmler, B. (2007) Diversity of the abundant pKLC102/PAGI-2 family of genomic islands in Pseudomonas aeruginosa. J Bacteriol 189: 2443-2459.

Luiten, R.G., Putterman, D.G., Schoenmakers, J.G., Konings, R.N., and Day, L.A. (1985) Nucleotide sequence of the genome of Pf3, an IncP-1 plasmid-specific filamentous bacteriophage of Pseudomonas aeruginosa. J Virol 56: 268-276.

McKew, B.A., Coulon, F., Osborn, A.M., Timmis, K.N., and McGenity, T.J. (2007a) Determining the identity and roles of oil-metabolizing marine bacteria from the Thames estuary, UK. Environ Microbiol 9: 165-176. 
McKew, B.A., Coulon, F., Yakimov, M.M., Denaro, R., Genovese, M., Smith, C.J., et al. (2007b) Efficacy of intervention strategies for bioremediation of crude oil in marine systems and effects on indigenous hydrocarbonoclastic bacteria. Environ Microbiol 9: 1562-1571.

Nelson, K.E., Weinel, C., Paulsen, I.T., Dodson, R.J., Hilbert, H., Martins dos Santos, V.A., et al. (2002) Complete genome sequence and comparative analysis of the metabolically versatile Pseudomonas putida KT2440. Environ Microbiol 4: 799-808.

Ornstein, R., Rein, R., Breen, D., and MacElroy, R. (1978) An optimized potential function for the calculation of nucleic acid interaction energies. Biopolymers 17: $2341-2360$.

Pedersen, A.G., Jensen, L.J., Brunak, S., Staerfeldt, H.H., and Ussery, D.W . (2000) A DNA structural atlas for Escherichia coli. J Mol Biol 299: 907-930.

Pride, D.T., Meinersmann, R.J., Wassenaar, T.M., and Blaser, M.J. (2003) Evolutionary implications of microbial genome tetranucleotide frequency biases. Genome Res 13: 145-158.

Reva, O.N., and Tümmler, B. (2004) Global features of sequences of bacterial chromosomes, plasmids and phages revealed by analysis of oligonucleotide usage patterns. BMC Bioinformatics 5: 90.

Reva, O.N., and Tümmler, B. (2005) Differentiation of regions with atypical oligonucleotide composition in bacterial genomes. BMC Bioinformatics 6: 251.

Röling, W.F., Milner, M.G., Jones, D.M., Lee, K., Daniel, F., Swannell, R.J., et al. (2002) Robust hydrocarbon degradation and dynamics of bacterial communities during nutrient - enhanced oil spill bioremediation. Appl Environ Microbiol 68: 5537-5548.

Sabirova, J.S., Ferrer, M., Regenhardt, D., Timmis, K.N., and Golyshin, P.N. (2006) Proteomic insights into metabolic adaptations in Alcanivorax borkumensis induced by alkane utilization. J Bacteriol 188: 3763-3773.

Saitou, N., and Nei, M. (1987) The neighbor-joining method: a new method for reconstructing phylogenetic trees. Mol Biol Evol 4: 406-425.

Satchwell, S.C., Drew, H.R., and Travers, A.A. (1986) Sequence periodicities in chicken nucleosome core DNA. J Mol Biol 191: 659-675.

Schneiker, S., Martins dos Santos, V.A., Bartels, D., Bekel, T., Brecht, M., Buhrmester, J., et al. (2006) Genome sequence of the ubiquitous hydrocarbondegrading arine bacterium Alcanivorax borkumensis. Nat Biotechnol 24: 997-1004.

Sharp, P.M., and Li, W.H. (1986) Codon usage in regulatory genes in Escherichia coli does not reflect selection for 'rare' codons. Nucleic Acids Res 14: 7737-7749. 
Shpigelman, E.S., Trifonov, E.N., and olshoy, A. (1993) CURVATURE: software for the analysis of curved DNA. Comput Appl Biosci 9: 435-440.

Sicheritz-Ponten, T., and Andersson, S.G. (2001) A phylogenomic approach to microbial evolution. Nucleic Acids Res 29: 545-552.

Skovgaard, M., Jensen, L.J., Friis, C., Stærfeldt, H.H., Worning, P., Brunak, S., and Ussery, D.W. (2002) The atlas visualisation of genome-wide information. In Methods in Microbiology. Wren, B., and Dorrell, N. (eds). London, UK: Academic Press, pp. 49-63.

Smits, T.H., Balada, S.B., Witholt, B., and van Beilen, J.B. (2002) Functional analysis of alkane hydroxylases from gram-negative and gram-positive bacteria. $J$ Bacteriol 184: 1733-1742.

Spangenberg, C., Fislage, R., Römling, U., and Tümmler, B. (1997) Disrespectful type IV pilins. Mol Microbiol 25: 203- 204.

Stover, C.K., Pham, X.Q., Erwin, A.L., Mizoguchi, S.D., Warrener, P., Hickey, M.J., et al. (2000) Complete genome sequence of Pseudomonas aeruginosa PA01, an opportunistic pathogen. Nature 406: 959-964.

Syutsubo, K., Kishira, H., and Harayama, S. (2001) Development of specific oliogonucleotide probes for the identification and in situ defection of hydrocarbon degrading Alcanivorax strains. Environ Microbiol 3: 371-379.

Teeling, H., Meyerdierks, A., Bauer, M., Amann, R., and Glockner, F.O. (2004) Application of tetranucleotide frequencies for the assignment of genomic fragments. Environ Microbiol 6: 938-947.

Ussery, D., Soumpasis, D.M., Brunak, S., Staerfeldt, H.H., Worning, P., and Krogh, A. (2002) Bias of purine stretches in sequenced chromosomes. Comput Chem 26: 531-541.

Wang, H., Noordewier, M., and Benham, C.J. (2004) Stress- Induced DNA Duplex destabilization (SIDD) in the E. coli genome: SIDD sites are closely associated with promoters. Genome Res 14: 15751584.

Weinel, C., Nelson, K.E., and Tümmler, B. (2002) Global features of the Pseudomonas putida KT2440 genome sequence. Environ Microbiol 4: 809-818. Willenbrock, H., and Ussery, D.W. (2007) Prediction of highly expressed genes in microbes based on chromatin accessibility. BMC Mol Biol 8: 11 .

Willenbrock, H., Friis, C., Juncker, A.S., and Ussery, D.W. (2006) An environmental signature for 323 microbial genomes based on codon adaptation indices. Genome Biol 7: R114. 
Worning, P., Jensen, .J., Hallin, P.F., Staerfeldt, H.H., and Ussery, D.W. (2006)

Origin of replication in circular prokaryotic chromosomes. Environ Microbiol 8: 353361.

Yakimov, M.M., Golyshin, P.N., Lang, S., Moore, E.R., Abraham, W.R., Lunsdorf, H., and Timmis, K.N. (1998) Alcanivorax borkumensis General nov., sp. nov., a new, hydrocarbon-degrading and surfactant-producing marine bacterium. Int J Syst

Bacteriol 48: 339-348. 